\title{
Synergistic Design of a Bio-Inspired Micro Aerial Vehicle with Articulated Wings
}

\author{
Jonathan Hoff* ${ }^{* \dagger}$, Alireza Ramezani*, Soon-Jo Chung* ${ }^{* \ddagger}$, and Seth Hutchinson*£ \\ ${ }^{*}$ Coordinated Science Laboratory, ${ }^{\dagger}$ Department of Mechanical Science and Engineering, \\ $\ddagger$ Department of Aerospace Engineering, ${ }^{\S}$ Department of Electrical and Computer Engineering \\ University of Illinois at Urbana-Champaign, Urbana, IL 61801 \\ Email: \{jehoff2, aramez, sjchung, seth\}@illinois.edu
}

\begin{abstract}
The sophisticated and intricate connection between bat morphology and flight capabilities makes it challenging to employ conventional flying robots to replicate the aerial locomotion of these creatures. In recent work, a bat inspired soft Micro Aerial Vehicle (MAV) called Bat Bot (B2) with five Degrees of Actuation (DoA) has been constructed to mimic the flight behavior of a biological bat. Major differences in structural topology resulted from this simpler kinematic complexity, and thus it is necessary to find the dimensions of B2's structure and the behavior of its actuators such that the wingbeat cycle of B2 closely mimics that of a biological bat. The current work assumes the previously designed structure of $\mathrm{B} 2$ and presents a synergistic design approach to imitate the kinematic synergies of a biological bat. Recent findings have unveiled that the most dominant synergies in a biological bat could be combined to accurately represent the original kinematic movement, therefore simplifying its dimensional complexity. In this work, Principal Component Analysis (PCA) has been employed in order to extract dominant principal components of biological bat flight kinematics. Thereafter, first and second principal components are chosen to shape the parametric kinematics and actuator trajectories of $\mathrm{B} 2$ through finite state nonlinear constrained optimization. The method yields a robot mechanism that despite having a few DoAs, it possesses several biologically meaningful morphing specializations.
\end{abstract}

\section{INTRODUCTION}

In recent years, design of bio-inspired robots based on the low dimensional representation of the kinematics of biological animals has gained attention. Using a similar design approach, this paper presents a methodology that builds upon recent work [12, 13, 14] by employing the fundamentals of kinematic synergies of a biological bat to shape the kinematic structure and wingbeat cycle of a bio-inspired bat robot called Bat Bot (B2). The topological structure of B2, a robotic Micro Aerial Vehicle (MAV) with bat morphology, is fundamentally different from that of a biological bat. Thus, optimization is necessary to find both the parameters for this given structure and the trajectories of the actuators to best match the kinematic behavior over a wingbeat of the biological bat. A constrained optimization routine is formulated that compares the synergies obtained from prerecorded trajectories of markers on a biological bat to equivalent simulated marker trajectories on B2. The performance functional consists jointly of the linear combination of sum of squared differences between Euclidean positions of the markers of the biological bat and B2 and the first two principal components resulting from Principal

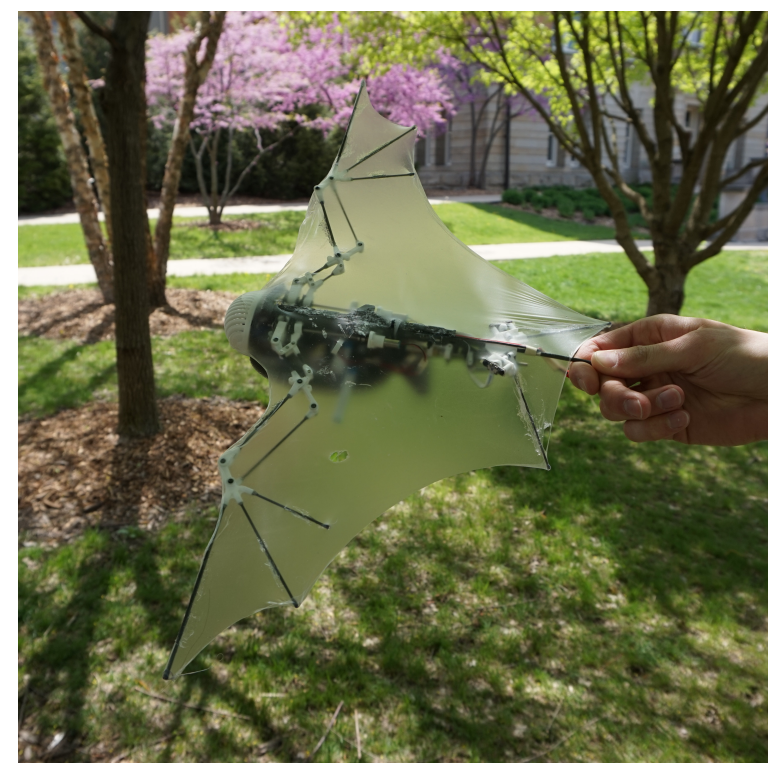

Fig. 1: B2 with optimized structure.

Component Analysis (PCA). These analyses synthesize the backbone design elements of B2's articulated flight mechanism and provide insight to the reference inputs to the motors powering its flapping flight such that B2 mimics the behavior of a biological bat.

This procedure requires consideration of past bio-inspired robots that applied fundamentally similar design approaches. The efforts in quantifying complex behavior of biological mechanisms in a lower dimensional subspace has led to the successful design of bio-inspired robots that can mimic their biological counterparts to a great extent [1, 3, 7, 9, 19]. Bioinspired robots are sometimes designed to take advantage of features in animals that are called muscle synergies, or unified activations within groupings of muscles. This notion of synergies, first proposed by Bernstein [2], is based on the theory that it is very difficult for the central nervous system to independently control all of the joints of an animal simultaneously. Kinematic movements likewise have synergies as a result of these muscular synergies. These synergies often form a set of basis vectors where only the most dominant are needed to approximate the animal's movement. For example, 
one Degree of Freedom (DoF) in animals may correspond to the coordinated movement of multiple joints [2]. One DoF is not necessarily expressed only as one joint because often movements of joints are coupled to each other. As a result, many bio-inspired robots have been designed to replicate synergies found in animals in order to design a robot that accurately replicates the behavior of this animal in spite of retaining fewer DoFs.

Numerous studies have been performed to quantify the set of synergies of biological animals in order to characterize their behavior in a lower dimensional space. Many have used the statistical method PCA to reduce the dimensions of a data set [8]. Daffertshofer et al. [6] demonstrated that by taking only a few of the principal components that account for most of the variance, the original data can be reconstructed fairly accurately. More specifically, PCA has been effective in decoding articulated skeleton in humans. The human hand has become a largely studied kinematic structure for synergies. It has been shown that greater than $80 \%$ of the variance of static grasping data could be described by the first two postural synergies, i.e. principal components [17]. This suggests that the data can be well approximated by linear combinations of these two principal components. Based on these findings, Brown and Asada [4] designed a robotic hand to embody the kinematic topology of a human hand but be actuated with only two motors controlling the first two postural synergies. Ciocarlie et al. [5] similarly expanded upon the analyses of [17] by presenting a method for grasp planning of robotic hands in the space spanned by the first two postural synergies.

Like the human hand, it is imperative to recognize the sophisticated complexity of the biological bat's flight apparatus. The apparatus possesses ball-and-socket and revolute joints that connect the bones and muscles to one another and synthesize a metamorphic musculoskeletal system with over 40 DoFs. It is known that some of these joints are passive while some are active [15]. Developing a bat-size MAV has several challenges and restrictions that have roots in weight, size, and power limitations [10, 11]. These restrictions motivate better understanding and selection of major DoFs in bats. Additionally, bat motion can be described in a low dimensional space using PCA. Riskin et al. [15] found that there are three groupings of joints in a bat wing that move together, accounting for 14 of 20 joints. This study also discovered that approximating the bat's motion with only one third of the principal components accounted for $95 \%$ of the variance of the original behavior. It should also be recognized that a bat wing has a very similar skeletal structure to that of a human hand. Though grasping movement is not usually periodic and bat motion is, the similarity of the bone structures and the success of synergistic design of robotic hands gives an optimistic perspective to this approach for B2's flight mechanism.

It can be seen that mimicking the kinematics of a biological bat is challenging because of its complex morphology. Implementing a bat's 40 DoFs as a robot would require a very large number of actuators. Given the strict weight

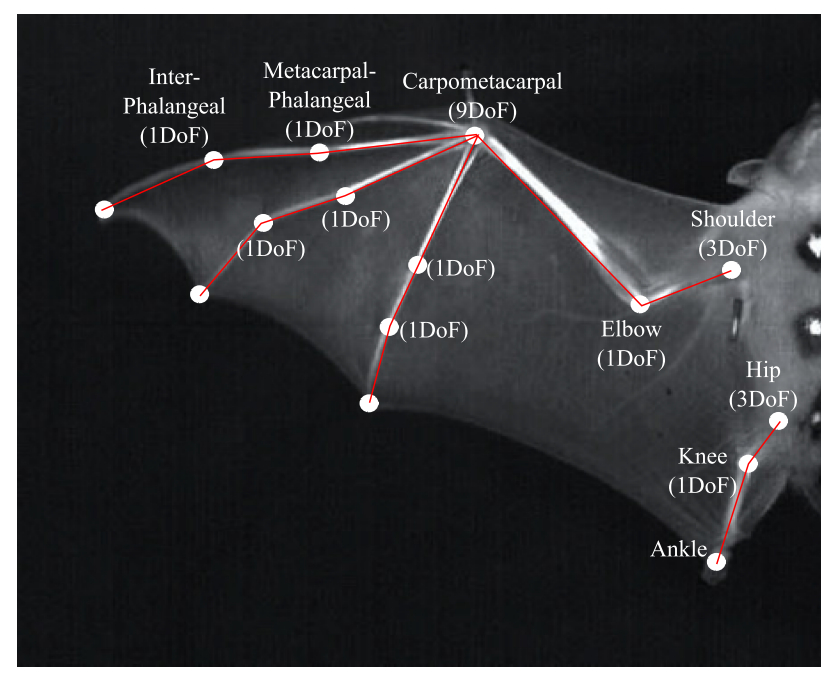

Fig. 2: DoFs of a biological bat. In producing this figure, an image from Riskin et al. [15] is used.

requirements necessary for flapping flight and the current limitations of technology, it is essentially infeasible to do this. Simplifications are therefore required for flight to be possible. A synergistic design approach inspired by functional group joints in biological bats has been used to synthesize a kinematic topology for B2 that has a reduced dimensional complexity but retains similar behavior to the studied organism [14].

The current work expands upon recently published works [12, 13, 14] on nonlinear dynamic modeling, flight control design, and hardware development of B2 by presenting a methodology for optimizing the articulated kinematics of a wingbeat cycle of $\mathrm{B} 2$ for this previously designed structure. It relies on the fundamentals of PCA to minimize the differences between the two most dominant principal components of B2 and those of a biological bat. An optimizer minimizes the sum of squared differences between Euclidean positions of corresponding markers on a biological bat and B2 as well as that between eigenvectors from PCA such that B2 tracks the trajectories of the markers on the biological bat and acquires matching synergies. The result of this procedure gives the actuator trajectories over a wingbeat cycle and the parameters describing B2's structure. It is worth noting that the resulting kinematics is not guaranteed to yield stable flight dynamics, and thus closed-loop feedback is required [12, 13].

The work in this paper is organized as follows. Section [II describes the biological bat motion capture data which will be compared against the kinematic behavior of B2. The construction and capabilities of B2 are briefly outlined in Section III In Section IV] a parametric kinematic model of B2 is derived that expresses the markers' positions in terms of the optimization variables, i.e., the position of the actuated coordinates and the physical parameters of B2. Using this model, Section $\mathrm{V}$ presents the constrained optimization formulation that finds the actuator trajectories over the wingbeat cycle of 
B2 and its structural parameters by comparing prerecorded trajectories of markers on a biological bat to its equivalent marker trajectories. The optimization results are recorded in Section VI Future work is explained in Section VII and concluding remarks are made in Section VIII.

\section{Motion CAPTURE DATA}

The design of a MAV with bat morphology that emulates the synergistic behavior of a biological bat can be improved with analysis of data describing its kinematics over a wingbeat cycle. A recent study recorded bats in a wind tunnel with markers painted on joints of their wings and in key locations necessary for correctly describing their motion [15]. The kinematic data set consists of the Cartesian coordinates of these data points that were tracked by high speed cameras. There are $\mathrm{n}$ time samples for a wingbeat cycle of a bat, with each sample containing the Cartesian coordinates for $\mathrm{n}_{\mathrm{p}}=17$ data markers placed on the bat. The position vectors of the markers are in the form $\mathbf{p}_{i}=\left[\begin{array}{lll}x_{i} & y_{i} & z_{i}\end{array}\right]^{\top}$. The data matrix is formulated as

$$
\mathcal{M}=\left[\begin{array}{cccc}
\mathbf{p}_{1}\left(t_{1}\right)^{\top} & \mathbf{p}_{2}\left(t_{1}\right)^{\top} & \cdots & \mathbf{p}_{\mathrm{n}_{\mathrm{p}}}\left(t_{1}\right)^{\top} \\
\mathbf{p}_{1}\left(t_{2}\right)^{\top} & \mathbf{p}_{2}\left(t_{2}\right)^{\top} & \cdots & \mathbf{p}_{\mathrm{n}_{\mathrm{p}}}\left(t_{2}\right)^{\top} \\
\vdots & \vdots & \vdots & \vdots \\
\mathbf{p}_{1}\left(t_{\mathrm{n}}\right)^{\top} & \mathbf{p}_{2}\left(t_{\mathrm{n}}\right)^{\top} & \cdots & \mathbf{p}_{\mathrm{n}_{\mathrm{p}}}\left(t_{\mathrm{n}}\right)^{\top}
\end{array}\right]
$$

The term $\mathbf{p}_{i}\left(t_{j}\right)$ refers to Cartesian coordinate vector of marker $i$ at time sample $j$.

Of these 17 recorded markers, 8 were carefully selected by matching similarities in structure between $\mathrm{B} 2$ and the biological bat and identifying the most important points to accurately describe the motion, setting $n_{p}=8$. Fig. 2 and 3 show the layouts of the skeletal structures of the biological bat and B2. The markers located on the shoulder, elbow, wrist, three fingertips, hip, and ankle correspond well to these same features on B2. Table I provides the equivalences between the selected data markers on the biological bat labeled in Fig. 2 and the corresponding markers on B2 labeled in Fig. 3 .

It should be noted that Ramezani et al. [14] designed B2 based on the dimensions of Rousettus aegyptiacus. This bat is much larger bat than Tadarida brasiliensis, the bat used for this study. Thus, the data used for this experiment was scaled such that the humerus length matches that of Rousettus aegyptiacus.

\section{Robotic BAt OVERVIEW [12, 13, 14]}

The biological bat data will be used to optimize both the structure and actuator trajectories of B2. Thus, a brief description of the construction of B2 is provided to achieve a basic understanding of its structure in order to interpret the parametric representation and the optimization routine presented in the next sections. A more detailed explanation of the construction and capabilities of the initial prototype of B2 has been documented in the previous works [12, 13, 14].

It is not feasible to design a MAV to incorporate all of the Degrees of Actuation (DoA) present in a biological bat. Thus, B2 was originally designed based on recent biological findings

\begin{tabular}{cc} 
Biological Bat & B2 \\
\hline \hline Shoulder & $p_{0}$ \\
Elbow & $p_{2}$ \\
Carpometacarpal & $p_{5}$ \\
Fingertip I & $p_{11}$ \\
Fingertip II & $p_{12}$ \\
Fingertip III & $p_{13}$ \\
Hip & $p_{14}$ \\
Ankle & $p_{15}$ \\
\hline
\end{tabular}

TABLE I: Selected data markers.

[16, 18] that emphasize the existence of functional group joints in bats. Several mechanical linkages are incorporated in the articulated flight mechanism of B2 in order to synthesize a morphing structure that possesses five DoAs. This morphing mechanism requires minimum numbers of actuators, while at the same time it is capable of producing biologically meaningful movements. These motions include: synchronous flapping motion of the left and right forelimbs, asynchronous mediolateral motion of each wing, and asynchronous dorsoventral movement of each leg.

The structural design parameters of B2 are given by the links lengths and angle measures of its mechanisms. There are eight links composing each forelimb of B2: the carpal link ( $p_{5^{-}}$ $\left.p_{6}\right)$, the three digital links, the radial link $\left(p_{3}-p_{5}\right)$, the radial support link $\left(p_{6}-p_{7}\right)$, the humeral link $\left(p_{0}-p_{7}\right)$, and the humeral support link $\left(p_{3}-p_{4}\right)$ [14]. The humeral link is divided into three sections measuring $h_{1}, h_{2}$, and $h_{3}$. Its support consists of a drive link $h_{d}$ and a support link $h_{s}$. The two components of the radial link measure $r_{1}$ and $r_{2}$, and its support has a length of $r_{s}$.

The three fingers are secured to the carpal plate of length $c$. These are thin flexible carbon fiber tubes of lengths $d_{1}, d_{2}$, and $d_{3}$. The origin of each measures a distance of $r$ from the end of the carpal plate $p_{6}$. Unlike biological bats, the digits of B2 lack joints and active manipulation. The angles of these digits with respect to the carpal plate are fixed, measuring to be $\gamma_{1}, \gamma_{2}$, and $\gamma_{3}$.

The movement of B2's hindlimbs is relaxed to only dorsoventral movement because mediolateral movement is less dominant in biological bats. Additionally, B2's hindlimbs ( $p_{14^{-}}$ $p_{15}$ ) of length of $l$ are carbon fiber rods that lack the knee joints present in its biological counterpart. These rods are connected to 1-DoF revolute joints on its structure, allowing each hindlimb to move in a plane rotated $\gamma_{4}$ from the parasagittal plane. The body length between the shoulder and hip $\left(p_{0}-p_{14}\right)$ is $b$.

All of these length and angle measurement parameters are lumped into a single parameter vector $\mathcal{P}$. This vector gives the exact layout for the given topological structure of B2.

\section{Parametric Flight Kinematics}

The static structure of B2 is determined by the physical parameters vector, but the evolution of its kinematics requires characterizing the actuators that drive its motion over a wingbeat cycle. For the purposes of this study, only consideration 


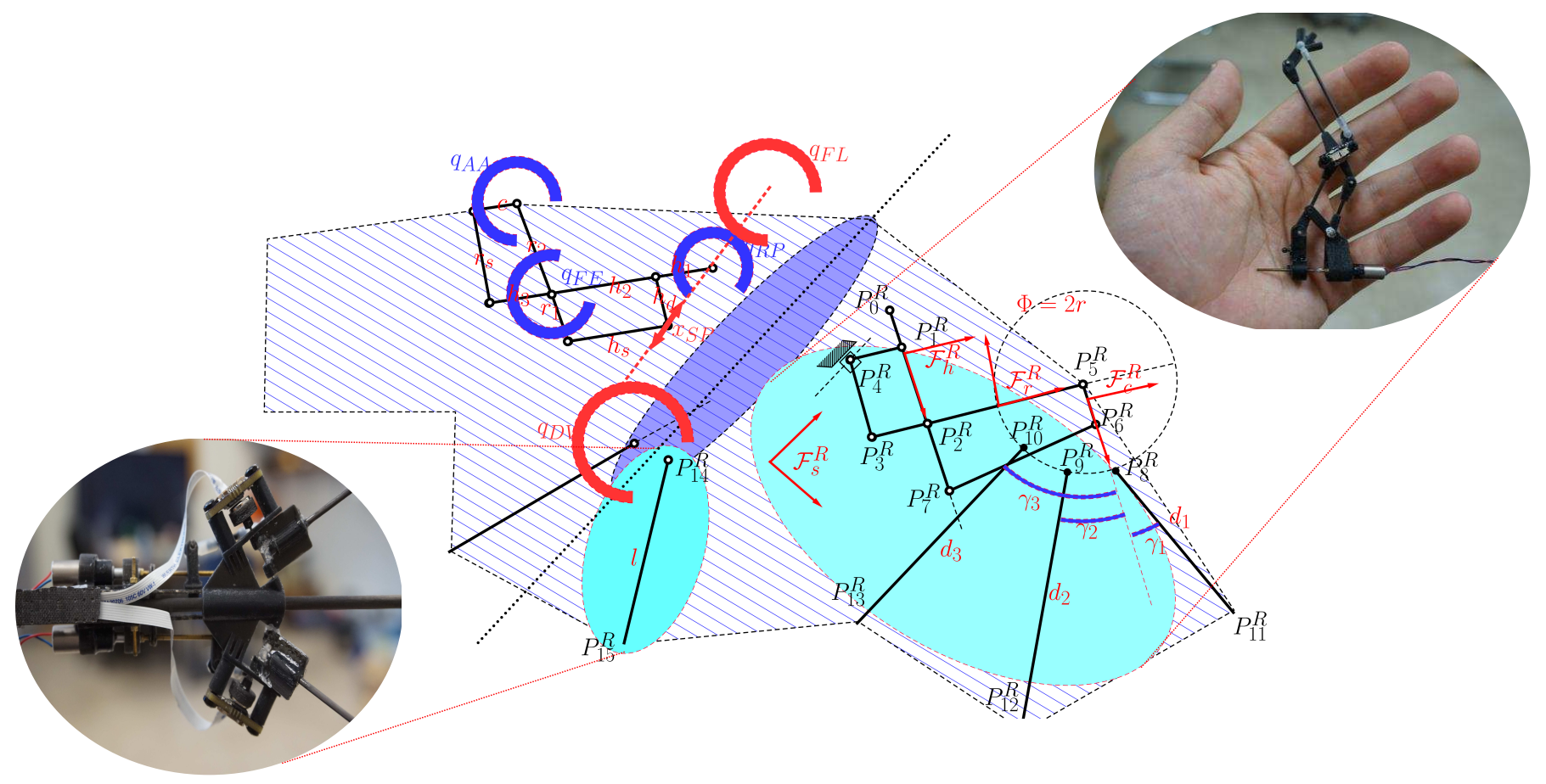

Fig. 3: DoFs and morphological parameters of B2 [14]. Blue circles denote biologically meaningful angles in the left forelimb that are not directly actuated. Red circles show directly actuated angles. Red indicates coordinate frames on the right wing. Black variables label the marker locations on the right wing, and red variables describe the structural parameters.

of one wing is necessary as it shall be assumed that the wings mirror each other in cruise flight. The actuated coordinates expressing the positions of the actuators are denoted by

$$
\mathcal{Q}_{\mathrm{a}}=\left[\begin{array}{lll}
x_{\mathrm{SP}} & q_{\mathrm{FL}} & q_{\mathrm{DV}}
\end{array}\right]^{\top}
$$

where $x_{\mathrm{SP}}$ is the position of the spindle $\left(p_{0}-p_{4}\right)$ that moves linearly to control the folding-unfolding of the wing; $q_{\mathrm{FL}}$ is the flapping angle and it is the angle that wing makes with respect to the $\mathbf{x}-\mathbf{y}$ plane; and $q_{\mathrm{DV}}$ characterizes the dorsoventral movement of the hindlimb.

The configuration variable vector, which defines the shape of the wing and hindlimb as it evolves through the action of actuated coordinates, is written

$$
\mathcal{Q}=\left[\begin{array}{llllll}
q_{\mathrm{RP}} & q_{\mathrm{FE}} & q_{\mathrm{AA}} & x_{\mathrm{SP}} & q_{\mathrm{FL}} & q_{\mathrm{DV}}
\end{array}\right]^{\top} .
$$

The configuration variable vector embodies five biologically meaningful DoFs. The angle $q_{\mathrm{RP}}$ describes the retraction and protraction angle, $q_{\mathrm{FE}}$ the radial flexion and extension angle, $q_{\mathrm{AA}}$ the abduction and adduction angle of the carpus, $q_{\mathrm{FL}}$ the flapping angle, and $q_{\mathrm{DV}}$ the dorsoventral movement of the hindlimb. This configuration variable vector together with the physical parameters vector yields the trajectories of the markers on B2.

The relationship between vectors $\mathcal{Q}$ and $\mathcal{P}$ and the marker trajectories is determined from the mechanical constraints of the mechanism of B2. The forelimb, shown in Fig. 3 is a sixbar linkage constrained to 1-DoF, which is known as the Watt mechanism [14]. Fundamentally, this consists of a three-link apparatus with two 1-DoF revolute joints attaching three links to each other with one of the links hinged to the body. The exact position of this mechanism can be described by three joint angles. Three links are added to constrain the motion to one DoF such that the position of the end-effector $p_{11}$ is determined by the position of one actuator. The carpal plate and humeral links attach to the radial support link with balland-socket joints, allowing for passive pronating movements. The humeral and humeral support links are connected with a link to replace the rotation of the humeral support with linear movements. Elbow flexion-extension is thereby generated from this linear motion of the humeral support, as the radial link's motion is dependent on that of the humeral link. Likewise, the digital links attached to the carpal plate move relative to the radial link.

Mathematically speaking, these mechanical constraints yield a nonlinear map

$$
\mathcal{G}_{\text {mech }}: \mathbb{R}^{3} \times \mathbb{R}^{19} \mapsto \mathbb{R}^{6} .
$$

This map takes actuated angles and physical parameters and it computes the configuration variable vector. From this, it is possible to compute marker trajectories. The shoulder coordinate frame $\mathcal{F}_{\mathrm{s}}$ has origin at $p_{0}$, and is aligned with the $\mathbf{x}$ and $\mathbf{y}$ axes as shown in Fig. 3 The humerus frame $\mathcal{F}_{\mathrm{h}}$ points along the humerus, the radius frame $\mathcal{F}_{\mathrm{r}}$ is set along the radial links, and the carpal frame $\mathcal{F}_{\mathrm{c}}$ is along the carpal plate. The hindlimb frame $\mathcal{F}_{1}$ is attached along the hindlimb. The numerical solutions satisfy the following system of nonlinear 
equations

$$
\left\{\begin{aligned}
& {\left[\hat{\mathbf{p}}_{i}\right]_{\mathcal{F}_{\mathrm{s}}=} } {\left[\hat{\mathbf{p}}_{0}\right]_{\mathcal{F}_{\mathrm{s}}}+R_{x}\left(q_{\mathrm{FL}}\right) R_{z}\left(q_{\mathrm{RP}}\right)\left[\hat{\mathbf{p}}_{i}\right]_{\mathcal{F}_{\mathrm{h}}}, } \\
& i \in\{1,2,7\} \\
& {\left[\hat{\mathbf{p}}_{i}\right]_{\mathcal{F}_{\mathrm{s}}}=} {\left[\hat{\mathbf{p}}_{2}\right]_{\mathcal{F}_{\mathrm{s}}}+R_{x}\left(q_{\mathrm{FL}}\right) R_{z}\left(q_{\mathrm{RP}}+q_{\mathrm{FE}}\right)\left[\hat{\mathbf{p}}_{i}\right]_{\mathcal{F}_{\mathrm{r}}}, } \\
& i \in\{3,5\} \\
& {\left[\hat{\mathbf{p}}_{4}\right]_{\mathcal{F}_{\mathrm{s}}=} } {\left[x_{\mathrm{SP}}, 0,0\right]^{\top} } \\
& {\left[\hat{\mathbf{p}}_{6}\right]_{\mathcal{F}_{\mathrm{s}}}=} {\left[\hat{\mathbf{p}}_{5}\right]_{\mathcal{F}_{\mathrm{s}}}+} \\
& R_{x}\left(q_{\mathrm{FL}}\right) R_{z}\left(q_{\mathrm{RP}}+q_{\mathrm{FE}}+q_{\mathrm{AA}}\right)\left[\hat{\mathbf{p}}_{6}\right]_{\mathcal{F}_{\mathrm{c}}} \\
& {\left[\hat{\mathbf{p}}_{i}\right]_{\mathcal{F}_{\mathrm{s}}=} } {\left[\hat{\mathbf{p}}_{6}\right]_{\mathcal{F}_{\mathrm{s}}}+} \\
& R_{x}\left(q_{\mathrm{FL}}\right) R_{z}\left(q_{\mathrm{RP}}+q_{\mathrm{FE}}+q_{\mathrm{AA}}+\gamma_{j}\right)\left[\hat{\mathbf{p}}_{i}\right]_{\mathcal{F}_{\mathrm{c}}}, \\
& i \in\{8,9,10,11,12,13\}, j \in\{1,2,3\} \\
& {\left[\hat{\mathbf{p}}_{15}\right]_{\mathcal{F}_{\mathrm{s}}=}\left[\hat{\mathbf{p}}_{14}\right]_{\mathcal{F}_{\mathrm{s}}}+R_{y}\left(q_{\mathrm{DV}}\right) R_{z}\left(\gamma_{4}\right)\left[\hat{\mathbf{p}}_{15}\right]_{\mathcal{F}_{1}} } \\
& {\left[\hat{\mathbf{p}}_{14}\right]_{\mathcal{F}_{\mathrm{s}}=}\left[\hat{\mathbf{p}}_{0}\right]_{\mathcal{F}_{\mathrm{s}}}+[-b, 0,0]^{\top} } \\
& R
\end{aligned}\right.
$$

where $R_{x}, R_{y}$, and $R_{z}$ are the rotation matrices with respect to the $\mathbf{x}, \mathbf{y}$, and $\mathbf{z}$ axes. The Cartesian position vectors of B2's markers with respect to the shoulder frame in the form $\left[\hat{\mathbf{p}}_{i}\right]_{\mathcal{F}_{\mathrm{s}}}=\left[\begin{array}{lll}\hat{x}_{i} & \hat{y}_{i} & \hat{z}_{i}\end{array}\right]^{\top}$ will for the remainder of this paper be expressed as $\hat{\mathbf{p}}_{i}$ to simplify notation. It should be noted that the position vector $\hat{\mathbf{p}}_{4}$ of spindle marker $p_{4}$ is restricted to motion along the $\mathbf{x}$ axis because of the constraints of the mechanism.

Furthermore, the geometric properties of B2's forelimb restrict it to have one DoA. This can be reflected mathematically by imposing constraints that the kinematics must satisfy. These emphasize that three loops made by $p_{0}-p_{1}-p_{4}, p_{1}-p_{2}-p_{3}-p_{4}$, and $p_{2}-p_{5}-p_{6}-p_{7}$, as shown in Fig. 3, are always closed. The end location of moving through the points in a given loop must be equal to the starting point, i.e. the links must stay connected. These constraints are explained by

$$
\left\{\begin{array}{l}
\| R_{z}\left(q_{\mathrm{RP}}\right)\left[\hat{\mathbf{p}}_{1}\right]_{\mathcal{F}_{\mathrm{h}}}+R_{z}\left(q_{\mathrm{RP}}+\psi\right)\left[-h_{d}, 0,0\right]^{\top} \\
-R_{z}\left(q_{\mathrm{RP}}\right)\left[\hat{\mathbf{p}}_{2}\right]_{\mathcal{F}_{\mathrm{h}}}-R_{z}\left(q_{\mathrm{RP}}+q_{\mathrm{FE}}\right)\left[\hat{\mathbf{p}}_{3}\right]_{\mathcal{F}_{\mathrm{r}}} \|_{2}^{2}=h_{s}^{2} \\
\| R_{z}\left(q_{\mathrm{RP}}\right)\left[\hat{\mathbf{p}}_{7}\right]_{\mathcal{F}_{\mathrm{h}}}-R_{z}\left(q_{\mathrm{RP}}\right)\left[\hat{\mathbf{p}}_{2}\right]_{\mathcal{F}_{\mathrm{h}}} \\
-R_{z}\left(q_{\mathrm{RP}}+q_{\mathrm{FE}}\right)\left[\hat{\mathbf{p}}_{5}\right]_{\mathcal{F}_{\mathrm{r}}} \\
-R_{z}\left(q_{\mathrm{RP}}+q_{\mathrm{FE}}+q_{\mathrm{AA}}\right)\left[\hat{\mathbf{p}}_{6}\right]_{\mathcal{F}_{\mathrm{c}}} \|_{2}^{2}=r_{s}^{2} \\
\left\|\left[\hat{\mathbf{p}}_{0}\right]_{\mathcal{F}_{\mathrm{s}}}+R_{z}\left(q_{\mathrm{RP}}\right)\left[\hat{\mathbf{p}}_{1}\right]_{\mathcal{F}_{\mathrm{h}}}-\left[\hat{\mathbf{p}}_{4}\right]_{\mathcal{F}_{\mathrm{s}}}\right\|_{2}^{2}=h_{d}^{2}
\end{array}\right.
$$

Additionally, the drive link $h_{d}$ was set to be the same length as $h_{1}$, and the two rectangles $p_{1}-p_{2}-p_{3}-p_{4}$ and $p_{2}-p_{5}-p_{6}-p_{7}$ were constrained to be parallelograms such that $h_{s}=h_{2}, r_{1}=h_{d}$, $r_{s}=r_{2}$, and $c=h_{3}$. Given these constraints, the kinematics of B2 can be shaped to match that of the biological bat.

\section{OPTIMIZATION}

The system of nonlinear equations given by (5) establishes the relationship of the configuration variables and physical parameters to the positions of the markers on B2 when the constraints (6) are satisfied. Using this map, the trajectories of the markers can be compared to those of the biological bat. This comparison is formulated as a constrained optimization problem in which the minimizing variables are the trajectories of the actuated coordinates $\mathcal{Q}_{\mathrm{a}}$ and the physical parameter vector $\mathcal{P}$. Each trajectory of $\mathcal{Q}_{\mathrm{a}}$ is approximated by an $\mathrm{n}_{\mathrm{a}}$ th order polynomial in the form

$$
a_{1} x^{\mathrm{n}_{\mathrm{a}}}+a_{2} x^{\mathrm{n}_{\mathrm{a}}-1}+\cdots+a_{\mathrm{n}_{\mathrm{a}}} x+a_{\mathrm{n}_{\mathrm{a}}+1} .
$$

The vector $\mathcal{A}$ is set to contain the coefficients of the polynomials, and thus $\mathcal{A}$ is an optimized variable. The optimization of $\mathrm{B} 2$ over a wingbeat cycle is formulated as

$$
\left\{\begin{array}{cl}
\underset{\mathcal{A}, \mathcal{P}}{\operatorname{minimize}} & \mathcal{J}(\mathcal{A}, \mathcal{P})=\sum_{i=1}^{\mathrm{n}_{\mathrm{p}}} \sum_{t=t_{1}}^{t_{\mathrm{n}}}\left\|\hat{\mathbf{p}}_{i}(t)-\mathbf{p}_{i}(t)\right\|^{2} \\
& +\alpha \sum_{j=1}^{\mathrm{n}_{\mathrm{pc}}}\left\|\hat{\mathbf{v}}_{j}-\mathbf{v}_{j}\right\|^{2} \\
\text { subject to } & f_{1}: x_{\mathrm{min}}-x_{\mathrm{SP}} \leq 0 \\
& f_{2}: x_{\mathrm{SP}}-x_{\mathrm{max}} \leq 0 \\
& g_{1}: x_{\mathrm{SP}}\left(t_{1}\right)-x_{\mathrm{SP}}\left(t_{\mathrm{n}}\right)=0 \\
& g_{2}: q_{\mathrm{FL}}\left(t_{1}\right)-q_{\mathrm{FL}}\left(t_{\mathrm{n}}\right)=0 \\
& g_{3}: q_{\mathrm{DV}}\left(t_{1}\right)-q_{\mathrm{DV}}\left(t_{\mathrm{n}}\right)=0 \\
& l_{k} \leq \mathcal{P}_{k} \leq u_{k}, k=1, \ldots, 19 .
\end{array}\right.
$$

The summation includes time samples from $t=t_{1}, \ldots, t_{\mathrm{n}}$, i.e. the entire wingbeat cycle. The term $\hat{\mathbf{p}}_{i}(t)$ is the position vector of marker $i$ at time sample $t$ of $\mathrm{B} 2$, and it is determined from $(5)$. The vector $\mathbf{p}_{i}(t)$ is that of the biological bat from the motion capture data.

The matrix $\hat{\mathcal{M}}$ is in the same format as $\mathcal{M}$ as described in (1), and $\mathbf{p}_{i}$ is replaced with $\hat{\mathbf{p}}_{i}$. The terms $\hat{\mathbf{v}}_{j}$ and $\mathbf{v}_{j}$ are the $j$ th principal components obtained from performing PCA on the data matrices $\hat{\mathcal{M}}$ and $\mathcal{M}$. Singular Value Decomposition (SVD) is applied to $\mathcal{M}$ as

$$
\mathcal{M}=\mathcal{U} \boldsymbol{\Sigma} \mathcal{V}^{\top}
$$

The data matrices are formatted such that $\mathcal{V}$ contains the principal components. Thus, the $j$ th column of $\mathcal{V}$ is the component $\mathbf{v}_{j}$. The matrix $\hat{\mathcal{M}}$ is likewise decomposed, and $\hat{\mathbf{v}}_{j}$ is the $j$ th column of $\hat{\mathcal{V}}$.

The objective function $\mathcal{J}$ includes cost terms of the sum of squared differences between the Euclidean positions of the markers and between the principal components to allow for 
reference tracking of the markers and to embed the synergies found in the biological bat in B2. The weighting coefficient $\alpha$ adjusts the impact of the two cost terms in the objective function such that both tracking and matching of synergies can be achieved.

The inequality constraints $f_{1}$ and $f_{2}$ enforce the mechanical limitations of the folding-unfolding motion of B2's forelimb by restricting the spindle position $x_{\mathrm{SP}}$ to the range between $x_{\min }$ and $x_{\max }$. The equality constraints $g_{1}, g_{2}$, and $g_{3}$ are given to ensure the periodicity of the actuator trajectories. The term $\mathcal{P}_{k}$ refers to the $k$ th element of $\mathcal{P}$, and these elements are restricted to a range with the lower $l_{k}$ and upper bounds $u_{k}$ on the optimization of the structural parameters. This prohibits very large or very small values that would be infeasible for the production of these links of B2.

The optimization problem is separated into four routines. Each of the three sets of trajectory coefficients $\mathcal{A}$ and the structural parameters $\mathcal{P}$ are optimized individually using MATLAB's constrained optimization algorithm fmincon with the interior-point algorithm. In each iteration of a given routine, the set of equations in (5) is solved to get the marker positions of B2. PCA is then performed to acquire the first two principal components of the given iteration. Finally, the objective function $\mathcal{J}$ is computed from these values. The sequence of the four optimization routines is iterated until a converging solution is reached.

Through simulations, it was found that 5 th order polynomials sufficiently replicated the actuator behaviors without overparameterization. Repetitions of the optimization procedure also indicated that using more than only the two most dominant principal components was redundant, thus setting the term $n_{\mathrm{pc}}=2$.

\section{RESUlts}

The results of the optimization are given by the trajectories of the actuated coordinates $\mathcal{Q}_{\mathrm{a}}$ and the values for the physical parameter vector $\mathcal{P}$. The values found for the physical parameters are given by Table $\Pi$, and the results are compared to the initial guess in Fig. 8 The most significant changes are the placement and measurements of the three digits.

Similar to a biological bat in cruise flight, B2's resulting period includes unfolding and folding of the wings as well as the vertical flapping motion of the wings. There are only slight movements of the hindlimbs over the wingbeat cycle.

The performance of the optimization routine can be evaluated by the similarity of the resulting two dominant principal components in the biological bat and B2. These components are eigenvectors that contain the magnitudes of the Cartesian coordinates of the markers, and these basis vectors create a low dimensional subspace that the motion of the bat resides in, if its dimensions were reduced to this space. Fig. 6 displays the similarity between the two sets of principal components. The variable number refers to the Cartesian coordinate of a given marker, and the weight determines its value. Thus, a principal component gives the positions of all of the data markers, and a linear combination of all of the principal components
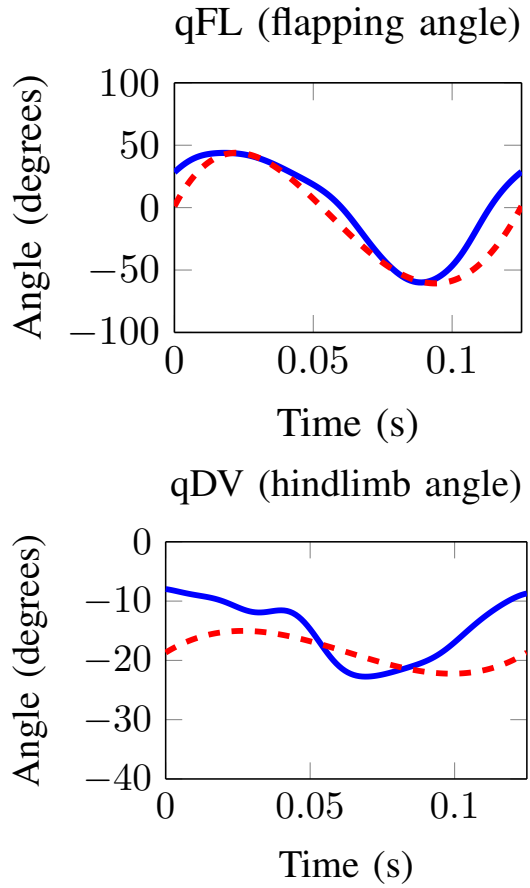

Fig. 5: Trajectory results from simulation of one wingbeat cycle of biologically meaningful angles of flapping and the hindlimb of B2 (dotted red) compared to those of a biological bat (blue). In B2, these angles are directly controlled by actuators.

reproduces the actual positions of the markers. Additionally, the marker trajectories can be projected onto these components to evaluate the importance of each over a wingbeat cycle. The similar behavior between B2 and the biological bat of these weights over a wingbeat is plotted in Fig. 7

The resulting evolutions of the five biologically meaningful angles of B2 over a wingbeat cycle were calculated via simulation and are compared to those of the biological bat in Fig. 4 and Fig. 5. Both have a flapping frequency of $8 \mathrm{~Hz}$, giving a wingbeat period of $0.125 \mathrm{~s}$. Only one wingbeat cycle is shown in the results. The angles of B2 track the trajectories of the angles of the biological bat, confirming the similarity in their behavior. The optimization formulation depended on matching dominant principal components, and the result gives matching joint angle trajectories between B2 and its biological counterpart. While B2 has a significantly reduced number of DoFs compared to a biological bat, there still exists a strong similarity between the progression of their joint angles over a wingbeat cycle. This validates the proposed synergistic optimization approach to finding the kinematic parameters for the constrained topology and actuator trajectories.

\section{FUTURE WORK}

Construction of B2 with the optimized parameters and actuator trajectories has begun and is shown in Fig. 1. however, stable closed-loop flight has not been achieved for this construction yet. Several challenges arose from hardware failure 

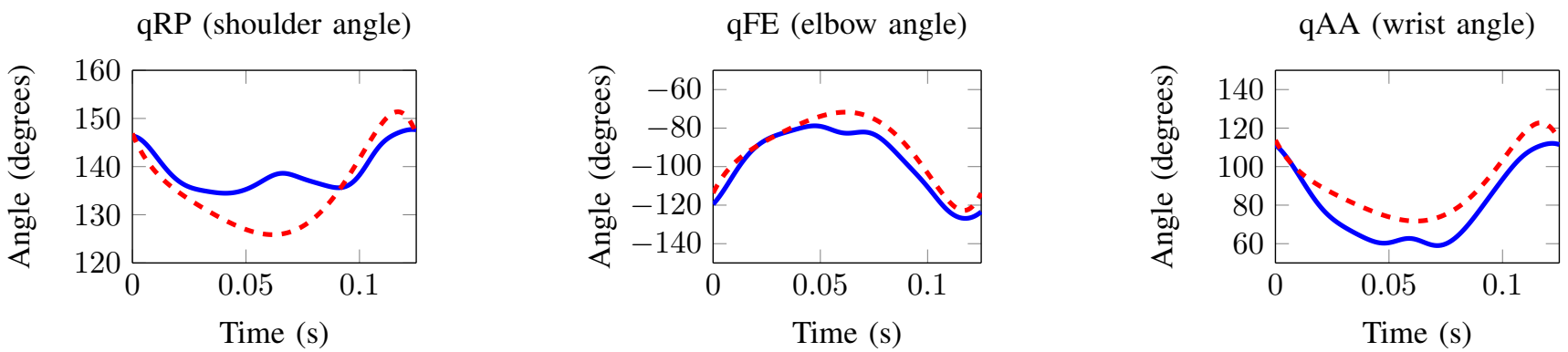

Fig. 4: Trajectory results from simulation of one wingbeat cycle of biologically meaningful angles in the forelimb of B2 (dotted red) compared to those of the biological bat (blue). These angles in B2 are not directly actuated as they move in response to the spindle $x_{\mathrm{SP}}$.

Principal component 1

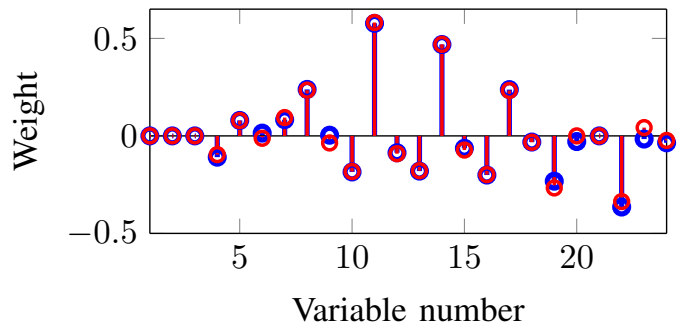

Principal component 2

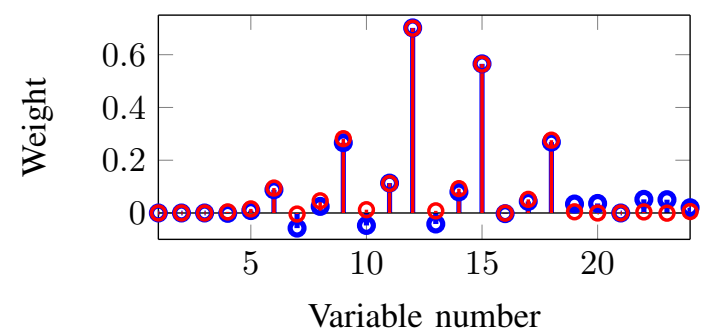

Fig. 6: Principal components 1 and 2 of the biological bat (blue) and B2 (red).

during testing. When executing the optimal actuator trajectories on the newly optimized structure, significant torques were generated, and these have caused physical failure of a number of components of the robot: 3D printed gears have shattered and the main drive shaft has been bent.

Furthermore, mechanical constraints and part limitations inhibited exact replication of the optimized structure given by Table II and this is identified as a potential reason for flight issues. First, actuator placement of the hindlimbs prevented a change in the body length $b$ of B2. Additionally, flight tests have shown that a hindlimb length of approximately $l=10 \mathrm{~cm}$ is necessary for stable cruise flight. Thus, the corresponding dimensions reported from the optimizer were not used in the construction. Three of the angles and two of the links of the optimized parameters were unable to be implemented on the new structure due to limitations on available parts. The angles
Projections of components 1 and 2

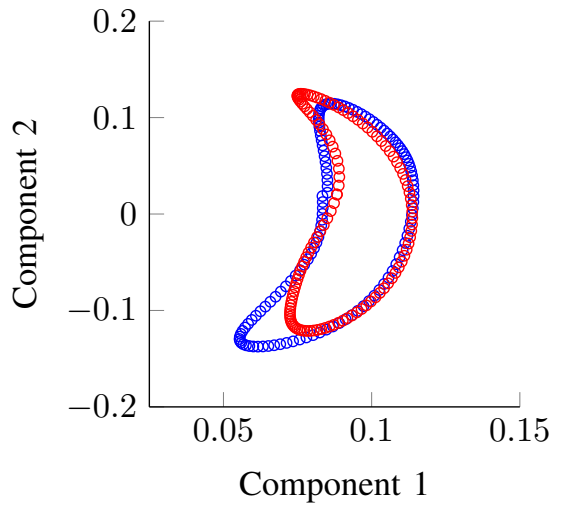

Fig. 7: Principal component projections 1 and 2 of the biological bat (blue) and B2 (red).

between the finger links, the third humeral link $h_{3}$, and the carpal plate $c$ were fixed to the old dimensions. Besides these restrictions, the forelimb mechanisms were constructed to fit the optimized parameters in Table $\Pi$.

Future testing of B2 with the optimal dimensions and actuator trajectories will ideally lead to stable flight. Addressing the failures in hardware and being able to use all of the optimized parameters will be important in succeeding. Fabricating more resilient components (e.g., a metal gear box) is now in progress, and it is hopeful that future flight tests will be successful.

\section{CONCLUSION}

A bat's morphology and dimensional complexity makes the design of a biomimetic MAV with equivalent complexity infeasible. Given the weight and power requirements necessary for flapping flight, it is currently too challenging to replicate this dimensional complexity with a robot. Thus, topological simplifications must be made to reduce this complexity while still retaining the morphological properties of the biological bat. B2 has been constructed with only five DoAs such that it exhibits synergies evident in biological bats. This paper adds to recent works on the development of B2 by presenting a 


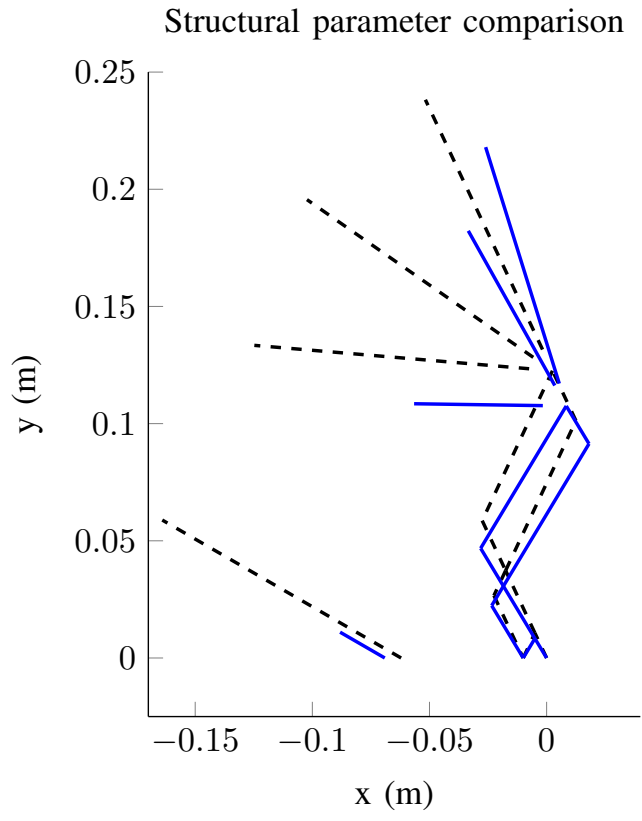

Fig. 8: Initial structural guess (dotted black line) of B2 vs. the resulting structure (blue line) after running optimization.

\begin{tabular}{ccc} 
Parameter & & Unit \\
\hline \hline$h_{1}$ & 1.0 & $\mathrm{~cm}$ \\
$h_{2}$ & 2.6 & $\mathrm{~cm}$ \\
$h_{3}$ & 1.9 & $\mathrm{~cm}$ \\
$h_{d}$ & 1.0 & $\mathrm{~cm}$ \\
$h_{s}$ & 2.6 & $\mathrm{~cm}$ \\
$r_{1}$ & 1.0 & $\mathrm{~cm}$ \\
$r_{2}$ & 7.1 & $\mathrm{~cm}$ \\
$r_{s}$ & 7.1 & $\mathrm{~cm}$ \\
$c$ & 1.9 & $\mathrm{~cm}$ \\
$r$ & 1.0 & $\mathrm{~cm}$ \\
$d_{1}$ & 10.6 & $\mathrm{~cm}$ \\
$d_{2}$ & 7.6 & $\mathrm{~cm}$ \\
$d_{3}$ & 5.5 & $\mathrm{~cm}$ \\
$b$ & 6.9 & $\mathrm{~cm}$ \\
$l$ & 2.2 & $\mathrm{~cm}$ \\
$\gamma_{1}$ & -13.7 & $\circ$ \\
$\gamma_{2}$ & -1.8 & $\circ$ \\
$\gamma_{3}$ & 58.1 & $\circ$ \\
$\gamma_{4}$ & 150.0 & $\circ$ \\
\hline
\end{tabular}

TABLE II: B2's morphological dimensions.

synergistic design method to further the development of the kinematic structure of B2 and shape its actuator trajectories over a wingbeat cycle. Further design has been implemented by applying kinematic optimization of these synergies to find the behavior of $\mathrm{B} 2$ over a wingbeat and the physical parameters defining the constrained topology.

This optimization routine achieves the kinematic structural parameters for the set topology of B2 and the trajectories of its actuators over a wingbeat. Minimizing the difference between the principal components of B2 and the biological bat consequently gives matching of their biologically meaningful angles. This result justifies the synergistic design approach outlined in this paper and confirms similar behavior of B2 to the biological bat through optimization of synergies. Though B2 has a significantly reduced dimensional complexity, it exhibits kinematic behavior like that of a biological bat.

It is worth noting that the resulting kinematics is not guaranteed to yield stable flight dynamics. This optimization design procedure is based solely on kinematic behavior; aerodynamic forces are not considered. Closed-loop feedback is necessary and is addressed in separate works. However, subsequent work can incorporate aerodynamic forces in the cost function to take into account flight dynamics.

\section{ACKNOWLEDGMENTS}

This research was supported by NSF Grant 1427111. The authors would like to thank the team of graduate and undergraduate students from aerospace, electrical, computer, and mechanical engineering departments at the University of Illinois at Urbana-Champaign for their contribution to construct the initial prototype of B2. Specifically, Thomas Smith and Zachary Curtis are acknowledged for assisting in the assembly of the robot with optimized dimensions.

The biological motion capture data set was provided by Kenneth Breuer and Sharon Swartz from Brown University. The authors would like to thank them in their assistance with this, as well as José Iriarte-Díaz for compiling the data.

The authors also appreciate the helpful insights and comments from the anonymous reviewers. Their feedback assisted in the improvement of this paper.

\section{REFERENCES}

[1] A. Ananthanarayanan, M. Azadi, and S. Kim. Towards a bio-inspired leg design for high-speed running. Bioinspiration \& Biomimetics, 7(4):046005, 2012.

[2] N. A. Bernstein. The coordination and regulation of movements. Pergamon Press, Oxford, 1967.

[3] M. C. Birch, R. D. Quinn, G. Hahm, S. M. Phillips, B. Drennan, A. Fife, H. Verma, and R. D. Beer. Design of a cricket microrobot In Proceedings of the IEEE International Conference on Robotics and Automation (ICRA), pages 1109-1114, 2000.

[4] C. Y. Brown and H. H. Asada. Inter-finger coordination and postural synergies in robot hands via mechanical implementation of principal components analysis In Proceedings of the IEEE/RSJ International Conference on Intelligent Robots and Systems (IROS), pages 2877 2882, 2007.

[5] M. Ciocarlie, C. Goldfeder, and P. Allen. Dimensionality reduction for hand-independent dexterous robotic grasping. In Proceedings of the IEEE/RSJ International Conference on Intelligent Robots and Systems (IROS), pages 3270-3275, 2007.

[6] A. Daffertshofer, C. J. C. Lamoth, O. G. Meijer, and P. J. Beek. PCA in studying coordination and variability: a tutorial Clinical Biomechanics, 19(4):415-428, 2004.

[7] C. Gong, M. J. Travers, H. C. Astley, L. Li, J. R. Mendelson, D. I. Goldman, and H. Choset. Kinematic 
gait synthesis for snake robots The International Journal of Robotics Research (IJRR), 35(1-3):100-113, 2016.

[8] I. Jolliffe. Principal component analysis John Wiley \& Sons, Ltd, 2002.

[9] G.-H. Liu, H.-Y. Lin, H.-Y. Lin, S.-T. Chen, and P.-C. Lin. A bio-inspired hopping kangaroo robot with an active tail Journal of Bionic Engineering, 11(4):541$555,2014$.

[10] D. J. Pines and F. Bohorquez. Challenges facing future micro-air-vehicle development AIAA Journal of Aircraft, 43(2):290-305, 2006.

[11] M. F. Platzer, K. D. Jones, J. Young, and J. C. S. Lai. Flapping wing aerodynamics: progress and challenges. AIAA Journal, 46(9):2136-2149, 2008.

[12] A. Ramezani, X. Shi, S.-J. Chung, and S. Hutchinson. Lagrangian modeling and flight control of articulatedwinged bat robot In Proceedings of the IEEE/RSJ International Conference on Intelligent Robots and Systems (IROS), pages 2867-2874, 2015.

[13] A. Ramezani, X. Shi, S.-J. Chung, and S. Hutchinson. Modeling and nonlinear flight controller synthesis of a bat-inspired Micro Aerial Vehicle In Proceedings of the AIAA Guidance, Navigation, and Control Conference, pages 2016-1376, 2016.

[14] A. Ramezani, X. Shi, S.-J. Chung, and S. Hutchinson. Bat Bot (B2), a biologically inspired flying machine. In Proceedings of the IEEE International Conference on Robotics and Automation (ICRA), 2016.

[15] D. K. Riskin, D. J. Willis, J. Iriarte-Díaz, T. L. Hedrick, M. Kostandov, J. Chen, D. H. Laidlaw, K. S. Breuer, and S. M. Swartz. Quantifying the complexity of bat wing kinematics. Journal of Theoretical Biology, 254(3):604615, 2008 .

[16] M. Rosén, G. R. Spedding, and A. Hedenström. The relationship between wingbeat kinematics and vortex wake of a thrush nightingale. Journal of Experimental Biology, 207(24):4255-4268, 2004.

[17] M. Santello, M. Flanders, and J. F. Soechting. Postural hand synergies for tool use The Journal of Neuroscience, 18(23):10105-10115, 1998.

[18] X. Tian, J. Iriarte-Díaz, K. Middleton, R. Galvao, E. Israeli, A. Roemer, A. Sullivan, A. Song, S. Swartz, and K. Breuer. Direct measurements of the kinematics and dynamics of bat flight. Bioinspiration \& Biomimetics, 1 (4):S10-S18, 2006.

[19] M. Wang, X. Zang, J. Fan, and J. Zhao. Biological jumping mechanism analysis and modeling for frog robot. Journal of Bionic Engineering, 5(3):181-188, 2008. 